\title{
Red Hawk Rocketry Boosted Dart
}

Akash Sen, Paul Ninneman, Katie VanderGalien, Jadee Kellogg, Robert Enright, Jimmy Izewski

\section{Abstract}

\author{
Ripon College
}

\begin{abstract}
As part of the Wisconsin Space Grant Consortium competition, Red Hawk Rocketry built a rocket for the Collegiate Rocket Launch Competition's non-engineering contest. The launch went well, albeit a little unstable,veering in the direction of the wind. This was unexpected because our RockSim model was considerably overstable, so it should have turned into the wind. Its apogee was around 1000 feet because our rocket was rather massive compared to other teams. However, because of its low apogee, we were able to easily locate and retrieve the rocket upon landing, as intended. Finally, all three aspects of the challenge were completed successfully; the dart separated, we were able to access mid-flight rotation data from our gyroscope, and we obtained on board, down looking video during the flight.
\end{abstract}

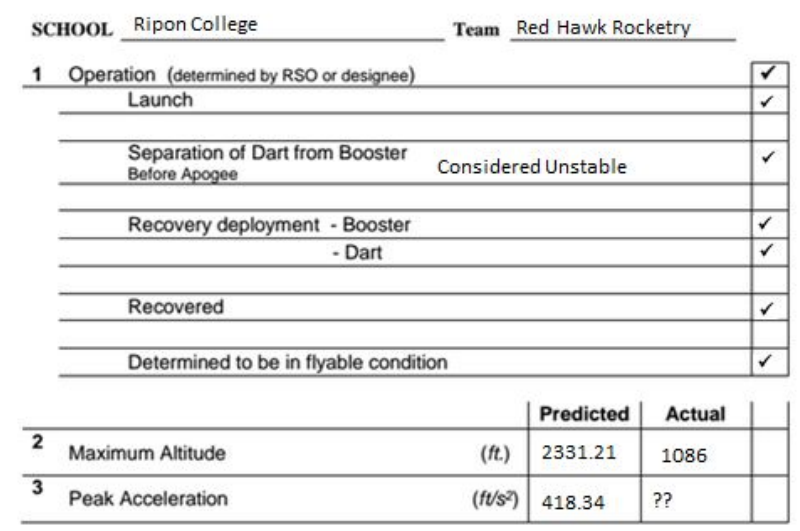

Figure 1: Flight performance comparison sheet showing information about the flight operations as well as the maximum altitude and separation.

\section{Performance Characteristics}

Operation characteristics were determined by a WSGC alliflate on the day of the launch. Maximum altitude and peak acceleration prediction was made by the rocket flight simulation program RockSim. The actual maximum altitude was taken by the competition altimeter, the Raven. Unfortunately, our team was unable to retrieve the peak acceleration from our altimeter due to electronic complications involving the wires required to connect to a computer. As seen below in Figure 1, our dart did separate from the booster, but the separation was considered unstable because the entire rocket tipped around 45 to about a second into the launch. The recovery devices for the dart and the booster both deployed as expected, and we were able to recover both parts of our boosted dart. 
Although our rocket was in flyable condition after retrieval, small tears in two of the couplers were noticed, but were mild enough that our rocket could still be flown. The damages sustained on these couplers were due to the selection of low quality components. We believe the damage may have been caused by a lashing of the shock cord when the parachutes deployed. Unfortunately, since we were not able to retrieve the acceleration data from our gyroscope, we could not form a comparison graph with the predicted data. However, Figure 2 has our predicted acceleration graph.

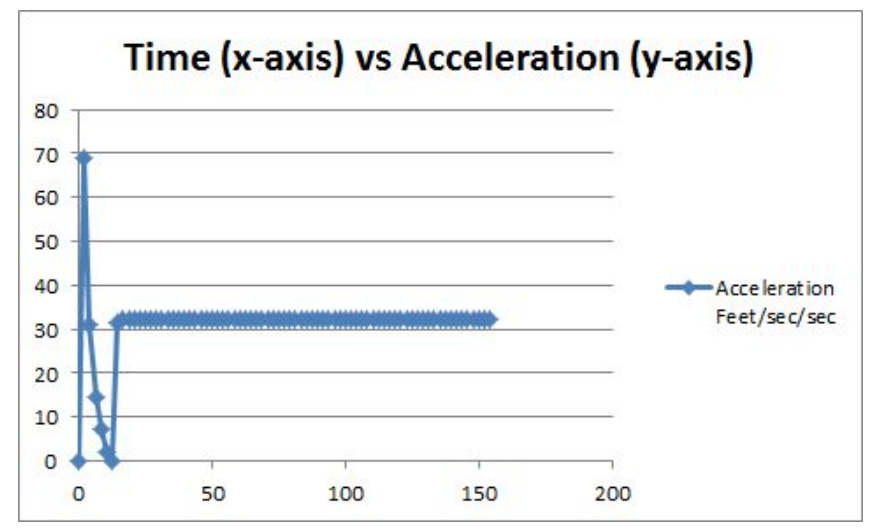

Figure 2: Predicted Acceleration Vs Time graph from RockSim

\section{Discussion and Results}

Our apogee was less than half of what we originally thought it would reach, based on the RockSim file. This could be due to factors like adding mass to the booster last minute to balance out the flight of the rocket, not taking into account a large increase in mass for both electronic bays compared to our theoretical RockSim launch, as well as weather conditions on the day of the launch. We found that the direction that the rocket flew upon launch to be unexpected. Even with the unaccounted added mass, the rocket should have been noticeably overstable, thus weathercocking into the wind. However, upon launch the rocket veered away from the wind instead of into it. One possible explanation could be one or more of the fins vibrating, causing instability. The fact that the rocket turned early, suggesting that it was not flying perpendicular to the ground and probably a leading cause of our lower than expected apogee. The dart did not sit in the booster at a slight angle from vertical. The dart was not set exactly perpendicular to the ground. This slight construction error may have caused some of the instability and curve in the launch. Due to the pressure of the situation and the speed required, it is possible that the wires were not set correctly, thus causing the rocket's engine to not burn evenly pushing it away from the wind.

Critical financial support was received by WSGC. 
An important note about the launch day is that our time on the pad had to be limited due to memory constrictions for the gyroscope data. We constructed switches out of wire that had to be twisted together and taped on the outside of the rocket to turn on all of our electronics. There was a total of four switches, which greatly increased the stress on the launch pad, especially with our time constraint. When we were ready to launch, there was no continuity for our rocket, and a launch assistant had to run back to the pad and reconnect the wires for the launch to take place. Although we had this small setback, we still had plenty of data from the gyroscope. For the future, we plan to buy physical switches to place in the rocket, so we don't have the added worry and time of twisting wires shortly before the launch.

The video captured by our internal camera was over 30 minutes long due to the fact it waited so long on the launch pad before connecting the switch to turn on the gyroscope. The camera had to be turned on remotely and placed inside the boat tail to record video. The camera was then secured into place using a bulkhead and screws and a piece of cloth was wrapped around it to limit movement. It was not until near the end of the video that the footage of the launch was recorded. Since our camera was placed inside the boat tail and looking down into the booster, the footage was completely black until separation. However, we were able to hear the countdown and the launch while the camera was filming the inside of the booster. After the dart separation occurred, the film became completely white until the camera adjusted for the light difference. Figure 3 shows a still image of the initial separation, which is still directed towards the inside of the booster.

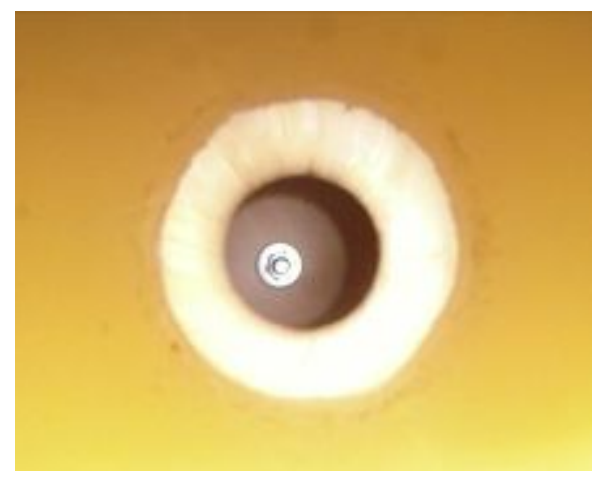

Figure 3: A picture of the dart at the first point of separation from the booster.

More screenshots of the footage can be seen below in Figures 5-7. Figure 5 appears to be the dart's apogee, Figure 6 is showing the moment when the parachute comes out, and Figure 7 is a photo of the ground when the dart is descending. 


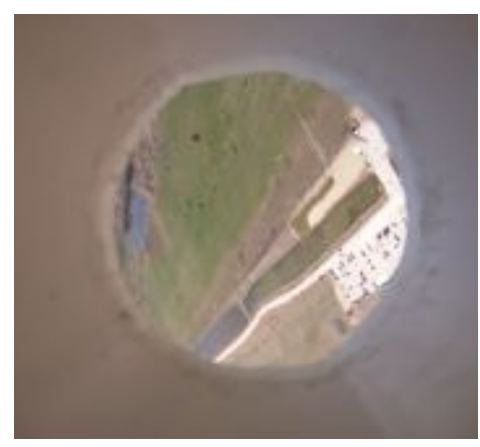

Figure 5: The ground as depicted from apogee

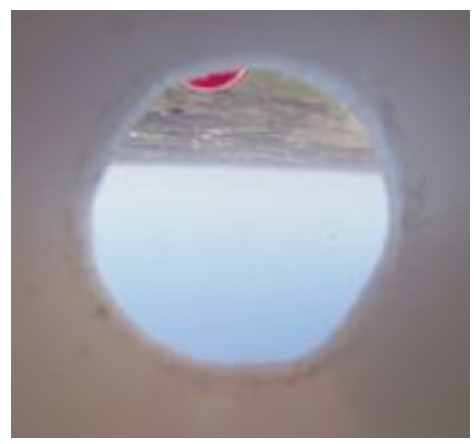

Figure 6: Picture of the dart jostling around due to the parachute ejection, the red spot being the parachute

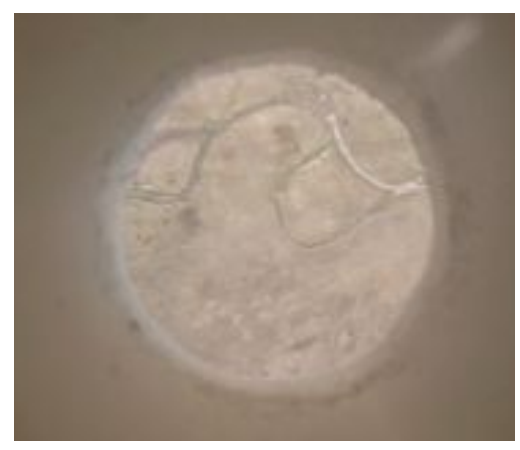

Figure 7: View from the bottom of the boattail when descending down to the ground.

The white ring around our video is the boat tail, as we could not fit the camera down further to get a wider range of view without taking off too much of the boat tail. The ground looks more curved due to the camera having a fisheye lens. We were very happy with the success of our on board camera, as it allowed us to compare the rotation measured by the gyroscope.

Our method of measuring the rotation of the dart using the $13 \mathrm{~g} 4200 \mathrm{~d}$ triple axis gyroscope breakout was successful. In order to properly visualize the $\mathrm{x}, \mathrm{y}$, and $\mathrm{z}$ data points that the gyroscope recorded, we needed to modify a few points. This is due to the fact that once the dart rotated around, the degrees looped from 0 to 360 , which meant that the low numbers corresponded to the high numbers. It was clear from random spikes where this occurred and we simply bumped those numbers back down. Once this was done we were able to use Excel to create a scatter plot of each coordinate versus time. The graphs for the $\mathrm{x}, \mathrm{y}$, and $\mathrm{z}$ versus time are shown below in Figures 9-11. It is important to note that our Arduino was set to record approximately 5 data points per second.

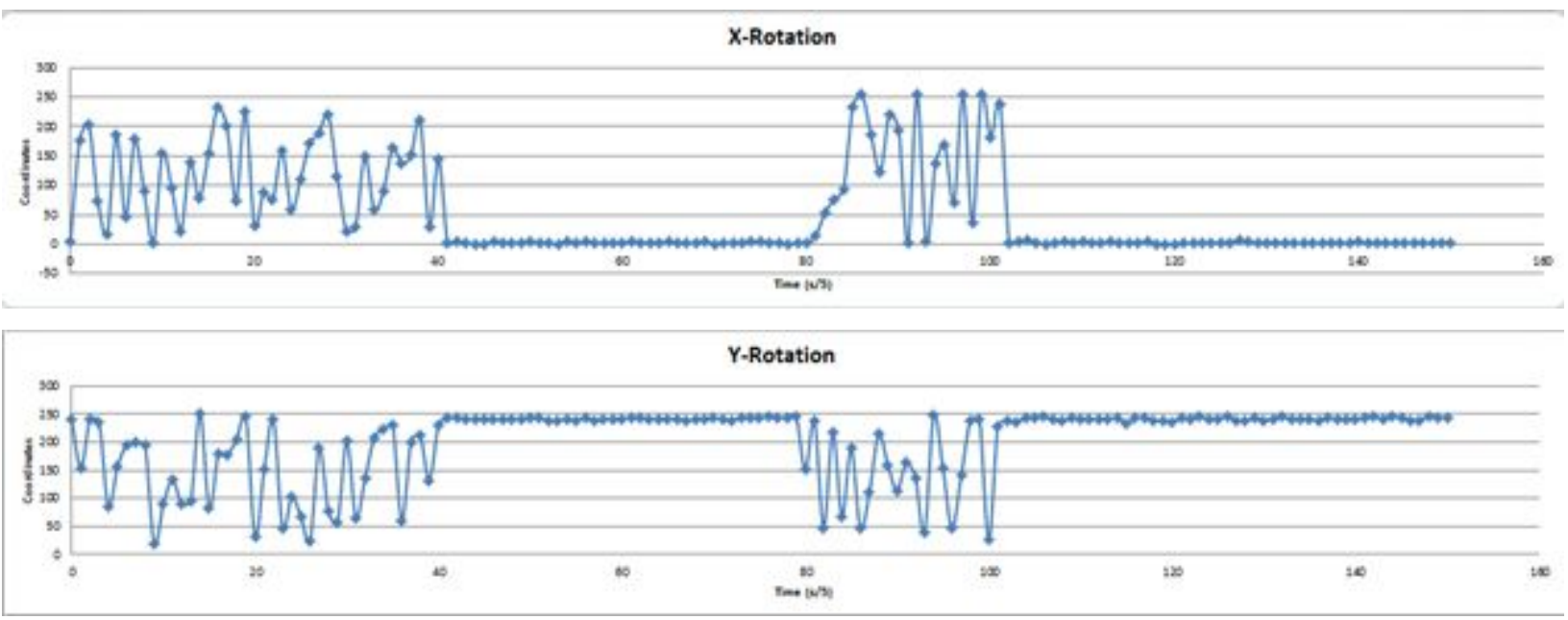

Critical financial support was received by WSGC. 
Figure 10: Degrees of rotation of our dart vs time in the y-direction

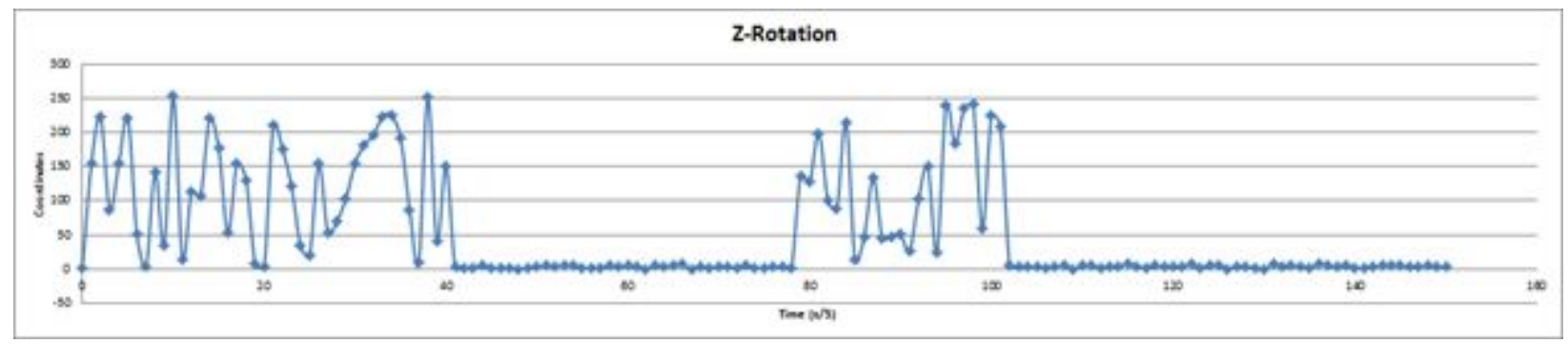

Figure 11: Degrees of rotation of our dart vs time in the z-direction

Here we can see that the $\mathrm{z}$ orientation is orientated vertically. If you look at the graph, we are able to interpret sections of the launch based off this knowledge. From the time of 0 to 5.4 seconds (which corresponds to the points 0 to 27 on the z-rotation graph), we are able to see our rocket weathercocking. Then from 5.4 seconds to 7 seconds (which corresponds to the points 27 to 35 on the graph), we are able to see that our dart clearly turns in one direction. This is confirmed from video footage, as we notice our dart veered mainly in the opposite direction that we had expected. From there we can see from 7.4 seconds to 8 seconds (corresponding to points 37 to 40), the parachute must deploy because of the sporadic movement of the dart. Once the parachute was fully deployed we can see that there is a clear period of time from 8.2 seconds to 15.4 seconds (points 41 to 77 ) in which there is a stable calm point for our dart. This area is clearly when our dart was descending to the ground. At 15.8 seconds to 20.2 seconds (corresponding to points 79 to 101) there is another section of sporadic movement. This is when the dart first hits the ground, and the parachute continues to pull it till both eventually settle down. The rest of our data points then settle down to a constant number for the remainder of the time. If we look at the $\mathrm{x}$ and the $\mathrm{y}$ rotation, we are able to conclude that the dart rotated approximately 12 times over 7.4 seconds before the parachute deployed. This means that it had a rotation rate of 1.6 rotations per second during that time frame.

\section{Conclusion}

Our rocket had a much lower apogee than expected, but because of this we were able to recover our rocket and have it deemed flyable. We believe the low apogee was caused by the rocket's weight which was more than $130 \mathrm{oz}$ and because the flight was not perfectly vertical. Our on board camera successfully filmed the separation of the dart and the booster, as well as the rest of the flight. We were also able to obtain data for the rotation of our dart in the $\mathrm{x}, \mathrm{y}$, and $\mathrm{z}$ directions over the course of its flight. Our rotation analysis from the gyroscope matched up with the footage from the camera. Overall, Red Hawk

Critical financial support was received by WSGC. 
Rocketry had a largely successful flight, completing all of the challenges for this year's competition. Red Hawk Rocketry would like to thank WSGC once again for this wonderful opportunity, and we look forward to competing again next year! 